В. Ц. Лыгденов, Ц. Гантулга, Н. Тувжаргал, Л. Энхтор. Оценка упругих постоянных $\gamma$-Fе из экспериментального фононного спектра

УДК 538.11

DOI 10.18101/2306-2363-2020-1-11-14

\title{
ОЦЕНКА УПРУГИХ ПОСТОЯННЫХ $\gamma$-Ге ИЗ ЭКСПЕРИМЕНТАЛЬНОГО ФОНОННОГО СПЕКТРА
}

() Лыгденов В. Ц.

научный сотрудник,

Бурятский государственный университет имени Доржи Банзарова

Россия, 670000, г. Улан-Удэ, ул. Смолина, 24а

Институт физического материаловедения СО РАН

Россия, 670031, г. Улан-Удэ, ул. Сахьяновой, 8

E-mail: lygdenov65@mail.ru

(С) Гантулга Ц.

кандидат физико-математических наук,

Монгольский государственный университет

Монголия, 210646, г. Улан-Батор, район Сухэ-Батор

E-mail: gantulga@smcs.num.edu.mn

(C) Тувжаргал Н.

кандидат физико-математических наук,

Монгольский государственный университет

Монголия, 210646, г. Улан-Батор, район Сухэ-Батор

E-mail: tuvjargal@num.edu.mn

\section{(c) Энхтор Л.}

кандидат физико-математических наук,

Монгольский государственный университет

Монголия, 210646, г. Улан-Батор, район Сухэ-Батор,

E-mail: enkhtor@num.edu.mn

Рассчитаны значения упругих постоянных $\gamma$-Fe при 1428 К из фононного спектра с применением методики описания экспериментального спектра металла с гранецентрированной кубической структурой. Значения упругих постоянных получены в длинноволновом приближении из фононных мод с применением трех интерпланарных силовых постоянных. Расчеты проводились методом наименьших квадратов путем описания экспериментальных фононных ветвей с помощью динамической матрицы, которая построена в модели Де Лане. Для проверки достоверности полученных значений упругих постоянных с их применением рассчитаны фононные ветви при сопоставлении с экспериментальными данными. Фононные ветви, рассчитанные с применением полученных в работе значений упругих постоянных, удовлетворительно описывают экспериментальный фононный спектр.

Ключевые слова: металл железо; фононный спектр; упругие постоянные; модель Де Лане; интерпланарные силовые постоянные; динамическая матрица; гранецентрированная кубическая структура.

Для цитирования: Лыгденов В. Ц., Гантулга Ц., Тувжаргал Н., Энхтор Л. Оценка упругих постоянных $\gamma$-Fe из экспериментального фононного спектра // Вестник Бурятского государственного университета. Химия. Физика. 2020. Вып. 1. С. 11-14. 
В [1] методом неупругого рассеяния нейтронов был измерен фононный спектр $\gamma$-Fe в направлениях симметрии при температуре 1428 К и описан с учетом силовых постоянных Борна-Кармана на первых шести координационных сферах, с применением которых рассчитаны значения упругих постоянных $\gamma$-Fe (таблица). Значения последних в литературе цитируются как упругие постоянные $\gamma$-Fе ввиду того, что упругие постоянные железа в $\gamma$-фазе не измерены методом ультразвуковых волн. Однако с учетом температурных зависимостей упругих постоянных $\mathrm{Fe}$, измеренных в [2] методом ультразвуковых волн в интервале температур $298 \div 1173$ К, можно заключить,что значения упругих постоянных $C_{11}$ и $C_{12}$, рассчитанные в [1] из фононного спектра, несколько завышены. В таблице даны значения упругих постоянных $\alpha$-Fe при 1428 К, которые получены экстраполяцией температурных зависимостей упругих постоянных, измеренных в [2]. Правомочность экстраполяции обоснована, установленным в [3], низким потенциальным барьером в Fе при переходе из $\alpha$ - фазы в $\gamma$-фазу. Из сравнения данных таблицы видно, что значения упругих постоянных $C_{11}, C_{12}$, рассчитанные в [1] из фононного спектра, превышают значения, которые оценены экстраполяцией данных [2]. Однако ввиду смягчения мод $\mathrm{T}_{1}[110]$ и $\mathrm{T}_{1}[112]$, установленного экспериментально в [3] при приближении к температуре фазового перехода $\alpha \rightarrow \gamma$, можно предположить, что упругие постоянные $\gamma$-Fe при 1428 К должны быть меньше значений для $\alpha-\mathrm{Fe}$, которые получены экстраполяцией из данных [2].

Упругие постоянные железа $\left(10^{10} \mathrm{H} / \mathrm{M}^{2}\right)$

Таблица

\begin{tabular}{|c|c|c|c|l|c|}
\hline & $C_{11}$ & $C_{12}$ & $C_{44}$ & \multicolumn{1}{c|}{ Ссылки и метод } & Температура \\
\hline$\gamma$-Fe & 15.4 & 12.2 & 7.7 & $\begin{array}{l}{[1] \text { из экспериментального фонон- }} \\
\text { ного спектра }\end{array}$ & $1428 \mathrm{~K}$ \\
\cline { 2 - 6 } & 13.1 & 10.3 & 7.5 & $\begin{array}{l}\text { данная работа из обработки дан- } \\
\text { ных [1] }\end{array}$ & $1428 \mathrm{~K}$ \\
\hline$\alpha-\mathrm{Fe}$ & 13.4 & 12.0 & 9.5 & Экстраполяция из данных [2] & $1428 \mathrm{~K}$ \\
\hline
\end{tabular}

Рассчитаны упругие постоянные $\gamma$-Fе при 1428 К из данных фононных дисперсий, измеренных в [2], с применением методоки описания экспериментального фононного спектра металла с гранецентрированной кубической структурой [4]. Значения упругих постоянных $\gamma$-Fe, которые приведены в табл. 1, получены нами в длинноволновом приближении из фононных мод L[100], T[100] и $\mathrm{T}_{1}[110]$ с применением трех интерпланарных силовых постоянных. Для проверки достоверности полученных значений упругих постоянных с их применением рассчитаны фононные ветви, которые показаны на рис. при сопоставлении с экспериментальными данными [1]. Из рисунка видно, что фононные ветви, рассчитанные с применением полученных в данной работе значений упругих постоянных, удовлетворительно описывают экспериментальный фононный спектр [1].

Из данных табл. видно, что полученное нами значение $C_{11}$ для $\gamma$-Fe близко к значению $C_{11}$, полученному из экстраполяции данных [2]. Значение $C_{44}$ находится в согласии с результатом [1]. Однако полученное в работе значение $C_{12}$ меньше результата [1] и отличается приблизительно на $20 \%$. 


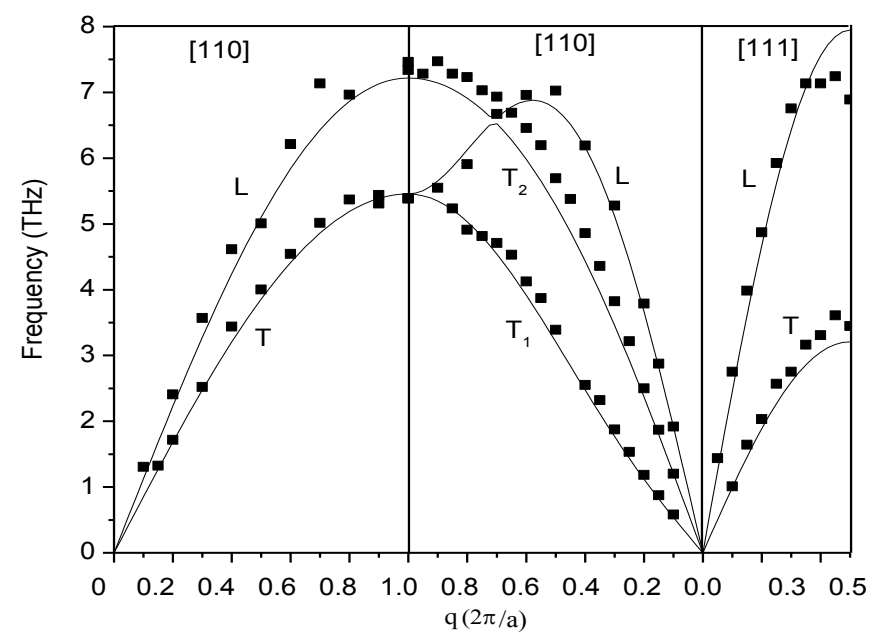

Рис. Фононный спектр $\gamma$-Fe при температуре 1480 К. Экспериментальные значения фононных частот [1] показаны квадратиками, а сплошные кривые соответствуют фононным ветвям, рассчитанным с применением полученных в данной работе значений упругих постоянных.

\section{Литература}

1. Zaretsky J., Stassis C. Lattice dynamics of $\gamma$-Fe // Phys. Rev. 1987. V.35. P. 4500-4512.

2. Dever D. J. Temperature dependence of the elastic constants in $\alpha$-iron single crystals: relationship to spin order and diffusion anomalies // J. Appl. Phys. 1972. V. 43. P. 3293-3299.

3. Neuhaus J., Petry W., Krimmel A. Phonon softening and martensitic transformation in $\alpha$ Fe // Physica B. 1997. V. 234-236. P. 897-907.

4. Энхтор Л., Силонов В. М. Расчет радиальных и тангенциальных силовых постоянных никеля из экспериментального фононного спектра // Вестник МГУ. 2016. № 1. C. 101-111.

\section{ESTIMATION OF ELASTIC CONSTANTS OF $\gamma$-Fe FROM EXPERIMENTAL PHONON SPECTRA}

Lygdenov $V$. Ts.

Research

Buryat State University,

67000, Russia, Ulan-Ude, Smolina, Str., 24a

Institute of Physical Materials Science SB RAS

670031, Russia, Ulan-Ude, Sakhyanovoy, Str., 6

E-mail: lygdenov65@mail.ru

\section{Gantulga Ts.}

Candidate of Physical and Mathematical Sciences National University of Mongolia

210646, Mongolia, Ulanbaatar, Sukhbaatar district

E-mail: gantulga@smcs.num.edu.mn 
Tuvjargal $N$.

Candidate of Physical and Mathematical Sciences

National University of Mongolia

210646, Mongolia, Ulanbaatar, Sukhbaatar district

E-mail: tuvjargal@num.edu.mn

Enkhtor L.

Candidate of Physical and Mathematical Sciences

National University of Mongolia

210646, Ulanbaatar, Mongolia, Sukhbaatar district

E-mail: enkhtor@num.edu.mn

The values of elastic constants $\gamma$-Fe at $1428 \mathrm{~K}$ from the phonon spectrum were calculated using the method of describing the experimental spectrum of a metal with a face-centered cubic structure. The values of elastic constants are obtained in the long-wave approximation from phonon modes using three interplanar force constants. The calculations were performed using the least squares method by describing experimental phonon branches using a dynamic matrix constructed in the De Lahn model. To verify the reliability of the obtained values of elastic constants with their application, phonon branches were calculated when compared with experimental data. The phonon branches calculated using the values of elastic constants obtained in the work satisfactorily describe the experimental phonon spectrum.

Keywords: metal iron; phonon spectrum; elastic constants; De Lahn model; interplanar force constants; dynamic matrix; face-centered cubic structure. 